\title{
FG-3019 anti-connective tissue growth factor monoclonal antibody: results of an open-label clinical trial in idiopathic pulmonary fibrosis
}

\author{
Ganesh Raghu1, Mary Beth Scholand ${ }^{2}$, João de Andrade ${ }^{3}$, Lisa Lancaster ${ }^{4}$, \\ Yolanda Mageto ${ }^{5}$, Jonathan Goldin ${ }^{6}$, Kevin K. Brown ${ }^{7}$, Kevin R. Flaherty ${ }^{8}$, \\ Mark Wencel $^{9}$, Jack Wanger ${ }^{10}$, Thomas Neff ${ }^{11}$, Frank Valone ${ }^{11}$, John Stauffer ${ }^{11}$ \\ and Seth Porter ${ }^{11}$
}

Affiliations: 'University of Washington, Seattle, WA, USA. ${ }^{2}$ University of Utah, Salt Lake City, UT, USA. ${ }^{3}$ University of Alabama at Birmingham, Birmingham, AL, USA. ${ }^{4}$ Vanderbilt University, Nashville, TN, USA. ${ }^{5}$ University of Vermont, Burlington, VT, USA. ${ }^{6}$ David Geffen School of Medicine, Los Angeles, CA, USA. ${ }^{7}$ National Jewish Health, Denver, CO, USA. ${ }^{8}$ University of Michigan Health System, USA. ${ }^{9}$ Via Christi Clinic, Wichita, KS, USA. ${ }^{10}$ Pulmonary Function and Clinical Trial Consultant, Rochester, MN, USA. ${ }^{11}$ FibroGen, Inc., San Francisco, CA, USA.

Correspondence: Ganesh Raghu, Division of Pulmonary and Critical Care Medicine, Campus Box 356522, Seattle, WA 98195-6522, USA. E-mail: graghuQu.washington.edu.

ABSTRACT FG-3019 is a fully human monoclonal antibody that interferes with the action of connective tissue growth factor, a central mediator in the pathogenesis of fibrosis.

This open-label phase 2 trial evaluated the safety and efficacy of two doses of FG-3019 administered by intravenous infusion every 3 weeks for 45 weeks in patients with idiopathic pulmonary fibrosis (IPF). Subjects had a diagnosis of IPF within the prior 5 years defined by either usual interstitial pneumonia (UIP) pattern on a recent high-resolution computed tomography (HRCT) scan, or a possible UIP pattern on HRCT scan and a recent surgical lung biopsy showing UIP pattern. Pulmonary function tests were performed every 12 weeks, and changes in the extent of pulmonary fibrosis were measured by quantitative HRCT scans performed at baseline and every 24 weeks.

FG-3019 was safe and well-tolerated in IPF patients participating in the study. Changes in fibrosis were correlated with changes in pulmonary function.

Further investigation of FG-3019 in IPF with a placebo-controlled clinical trial is warranted and is underway.

@ERSpublications

FG-3019 demonstrated good outcomes in changes in pulmonary function and extent of pulmonary fibrosis in IPF http://ow.ly/Xn7B4 


\section{Introduction}

Idiopathic pulmonary fibrosis (IPF) is a chronic, fatal lung disease of unknown aetiology, characterised by progressive dyspnoea and relentless loss of lung function [1]. The natural course of IPF, while variable over time, is universally progressive and the prognosis is poor with 5-year survival rates in the range of approximately $20-40 \%$ [2].

Connective tissue growth factor (CTGF) is an essential mediator of human fibrotic conditions [3] and represents a common pathway for fibrogenesis. When expressed in excess, CTGF co-ordinately upregulates growth factors such as transforming growth factor $\beta$ and insulin-like growth factor 1 . While normally expressed at low levels in healthy adults, excess CTGF has been shown to promote significant production of extracellular matrix (ECM) forms of both collagen and fibronectin, as well as inhibitors of metalloproteinases that normally inhibit breakdown of ECM components. The accumulation of ECM inherent in fibrosis is driven by both increased secretion and decreased degradation [3-5].

FG-3019 is a fully human monoclonal antibody specific for CTGF. Pre-clinical studies suggest that FG-3019 penetrates into tissues to reduce effective tissue levels of CTGF resulting in reduction of pro-fibrotic factors, enabling a rebalancing of ECM secretion and processing, and a return toward tissue homeostasis. In a mouse model of radiation-induced pulmonary fibrosis, administration of FG-3019 for 8 weeks beginning 16 weeks after lethal irradiation, when severe tissue remodelling was already advanced, resulted in altered gene expression in the lungs, reversal of lung pathology, decrease in abnormal lung density, abrogation of fibrosis, improvement in lung function and prolonged survival [6]. Notably, in irradiated animals, FG-3019 treatment altered the elevated expression of an array of fibrosis-related genes in the lungs, including $\geqslant 3$-fold reductions in expression levels of fibronectin, CTGF, lysyl oxidase and collagen $1 \alpha 1$.

In an open-label, phase 1b, dose-escalation study in 21 IPF patients, FG-3019 demonstrated no dose-limiting toxicity, dose-dependent adverse events (AEs) or drug-related serious adverse events (SAEs) [7]. Herein, we present data from a phase 2, prospective, open-label safety and efficacy study of FG-3019 in 89 subjects with IPF, and demonstrate good safety and tolerability, promising outcomes in changes of forced vital capacity (FVC) and a decrease in the extent of the radiographic pattern of pulmonary fibrosis in some patients.

\section{Methods}

Study design

Study FGCL-3019-049 was an open-label, single-arm, multicentre, phase 2a clinical trial conducted in the USA in subjects with IPF to evaluate the safety, tolerability and efficacy of FG-3019. Subjects received $15 \mathrm{mg} \cdot \mathrm{kg}^{-1}$ (cohort 1) or $30 \mathrm{mg} \cdot \mathrm{kg}^{-1}$ (cohort 2) of FG-3019 intravenously every 3 weeks over 45 weeks. For both cohorts, eligible subjects were $35-80$ years old at screening with a clinical diagnosis of IPF within the prior 5 years along with either usual interstitial pneumonia (UIP) pattern on a high-resolution computed tomography (HRCT) scan within 5 years of screening, or a possible UIP pattern on an available HRCT scan plus a recent surgical lung biopsy showing UIP pattern. The protocol stipulated HRCT evidence of $\geqslant 10 \%$ to $<50 \%$ parenchymal fibrosis (reticulation) and $<25 \%$ honeycombing within the whole lung. For cohort 1, inclusion criteria stipulated FVC of $45-85 \%$ of predicted value [8], diffusing capacity of the lung for carbon monoxide (DLCO) $\geqslant 30 \%$ predicted, objective evidence of disease progression (by either computed tomography (CT) scan, pulmonary function tests (PFTs) or dyspnoea) and decline in pulmonary function or worsening dyspnoea in the prior 3-12 months. For cohort 2, the FVC inclusion criterion was adjusted to $\geqslant 55 \%$ of predicted value, with evidence of IPF disease progression within preceding 18 months, defined as above.

Exclusion criteria for both cohorts included DLCO $<30 \%$ of the predicted value, corrected for haemoglobin; infiltrative lung disease other than IPF; acute exacerbation of IPF within the prior 3 months; forced expiratory volume in $1 \mathrm{~s} / \mathrm{FVC}$ ratio $<0.65$; extent of emphysema greater than the extent of fibrosis on HRCT; significant comorbid medical conditions; and concurrent treatment with immunomodulatory or immunosuppressive drugs.

Daily treatment with prednisone $(\leqslant 10 \mathrm{mg})$ and/or $N$-acetylcysteine $(\leqslant 1800 \mathrm{mg})$ was allowed provided that this treatment had been continuous for $\geqslant 4$ months prior to screening and was to be continued throughout the treatment period. Treatment of exacerbations of IPF at the discretion of the investigator (including use of corticosteroids) was acceptable for up to 4 weeks.

Efficacy end-points included PFT, HRCT and measures of health-related quality of life. An expert pulmonary physiology consultant certified each clinical site based on rigorous performance standards according to a study-specific PFT procedure manual and the consensus guidelines of the American Thoracic Society/European Respiratory Society [9]. All PFTs measured at baseline (BL) and every 12 weeks were read by an independent central reader. 
Visual reading of the screening HRCT scan by a central reader (MedQIA, Los Angeles, CA, USA) determined the eligibility of each subject. HRCT was performed using a standardised image acquisition protocol with careful attention to use of the same HRCT platform at each visit, and using a standardised acquisition technique and reconstruction algorithm. In order to standardise the lung volume at which images were obtained, HRCT technologists were trained to coach subjects to hold their breath as close to total lung capacity (TLC) as possible, which has previously been shown to be feasible [8]. Quality control was carefully monitored during the study, including confirmation of good correlation between the TLC measured by HRCT and the TLC measured by PFT to ensure the reproducibility of the breath-holding manoeuver. Both modalities of TLC assessment have been shown previously to be strongly correlated [10]. In this study, correlation values ( $r$ ) for TLC measured by HRCT and by PFT were 0.89 at both BL and week 48. For the correlation of PFT- and HRCT-based TLC changes from baseline to week 48, $\mathrm{r}=0.34$ $(p=0.006)$. Changes in the extent of fibrosis from screening were measured at 24 weeks and at 48 weeks ( 3 weeks after the last dose) using quantitative analysis of thoracic HRCT by the central reader with a $510 \mathrm{k}$-cleared computer algorithm (MedQIA) that provides an overall determination of the percentage of the lung that contains ground glass (GG), reticular fibrosis with architectural distortion (QLF), honeycomb fibrosis (HC), and a composite score that represents a summation of QLF, GG and HC (QILD) [11, 12].

Data in tables and figures are presented as observed values only. Where discussed in the results section, imputation included observed values plus imputed values for missing data based on mixed-model, repeated measures.

This study was performed in accordance with the Declaration of Helsinki. Ethics committees at each participating institution reviewed and approved the protocol before subjects were enrolled in the trial. Each subject was fully informed of the risks and benefits of participating in this trial, and provided written informed consent prior to screening (www.ClinicalTrials.gov number NCT01262001).

\section{Results}

\section{Subject information}

Between March, 2011, and December, 2012, 127 patients were screened and 90 subjects were enrolled in two cohorts, 89 of which received at least one dose of FG-3019 (figure 1). The study population was predominantly male and white, with a mean age of 67.9 years. Demographic data for each cohort are summarised in table 1. BL PFT and HRCT values are shown in tables 2 and 3 (BL PFT values represent a mean of values obtained at screening and on day 1 ). Mean BL FVC for cohort 1 was $62.8 \%$ predicted (range $42.6-89.6 \%$ predicted) and for cohort 2 it was $72.7 \%$ predicted (range $52.9-111.7 \%$ predicted). Mean BL DLCO for cohort 1 was $48.3 \%$ predicted (range $31.1-94.5 \%$ predicted) and for cohort 2, it was $49.7 \%$ predicted (range $30.4-89.6 \%$ predicted). 67 (75\%) subjects completed the study and 66 of these had week 48 PFTs and HRCT determinations. Inclusion criteria included evidence of IPF disease progression during the preceding 12 (cohort 1 ) or 18 months (cohort 2), defined as visually apparent worsening of abnormalities on HRCT or decline in FVC \% predicted value by $10 \%$ or more. These criteria were intended to enrich for patients with progressive rather than stable disease. The change of inclusion criteria between cohort 1 and cohort 2 was based on two observations. In cohort 1, subjects with BL FVC $<55 \%$ predicted generally did poorly in the trial with regard to changes in pulmonary function and changes in fibrosis. Of the 22 subjects who prematurely discontinued from the study, 13 had a BL FVC $<55 \%$ predicted. In addition, the criteria for evidence of progressive disease within 3-12 months proved to be unduly restrictive and so were loosened to 18 months in the second cohort. As a pilot study precursor to a more robust placebo-controlled trial, these adjustments seemed prudent.

Our hypothesis was that FG-3019 treatment could reverse parenchymal fibrosis. Consequently, the inclusion criteria stipulated an extent of whole-lung parenchymal fibrosis $(\geqslant 10 \%$ to $<50 \%$ ), assuming that some fraction of that fibrosis could be reversible, with no more than $25 \% \mathrm{HC}$, which was assumed to be irreversible.

\section{Safety}

Safety outcomes are summarised for each cohort in table 4. 24 (27.0\%) out of 89 treated subjects experienced a total of 38 treatment-emergent SAEs during the trial. 13 (54\%) of the 24 subjects with SAEs dropped out of the study before the week 48 time-point, and 24 of the 38 SAEs occurred in those 13 subjects. SAEs in 11 subjects were respiratory in nature, the most common of which were pneumonia (nine subjects), IPF exacerbation (six subjects) and respiratory failure (three subjects). SAEs in 12 subjects were nonrespiratory in nature, which reflected the underlying comorbid conditions known to be associated with this patient population (e.g. cerebrovascular accident, hypotension, major depression and transient ischaemic attack), with no clear trends. Of the 22 subjects who prematurely discontinued the study, 13 (59\%) had a BL FVC <55\% predicted. Eight deaths occurred during the trial, including three from respiratory failure, and one each for end-stage IPF, worsening IPF, IPF exacerbation, pneumonia and acute 


\section{Cohort 1 (15 mg.kg-1 FG-3019)}

IPF for $\leqslant 5$ years

FVC $45-85 \%$ predicted, $D\llcorner C 0 \geqslant 30 \%$ predicted Lung fibrosis $\geqslant 10 \%$ to $<50 \%, \leqslant 25 \%$ honeycomb Disease progression in previous 12 months 72 patients screened

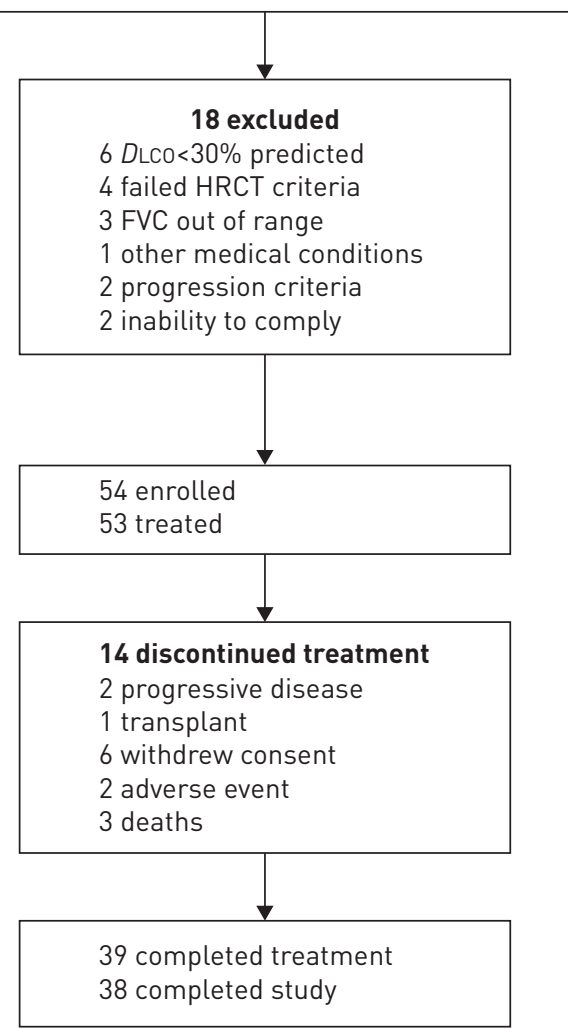

Cohort 2 (30 mg $\left.\cdot \mathrm{kg}^{-1} \mathrm{FG}-3019\right)$

IPF for $\leqslant 5$ years

FVC $\geqslant 55 \%$ predicted, $D\llcorner C 0 \geqslant 30 \%$ predicted Lung fibrosis $\geqslant 10 \%$ to $<50 \%$, $\leqslant 25 \%$ honeycomb Disease progression in previous 18 months 55 patients screened

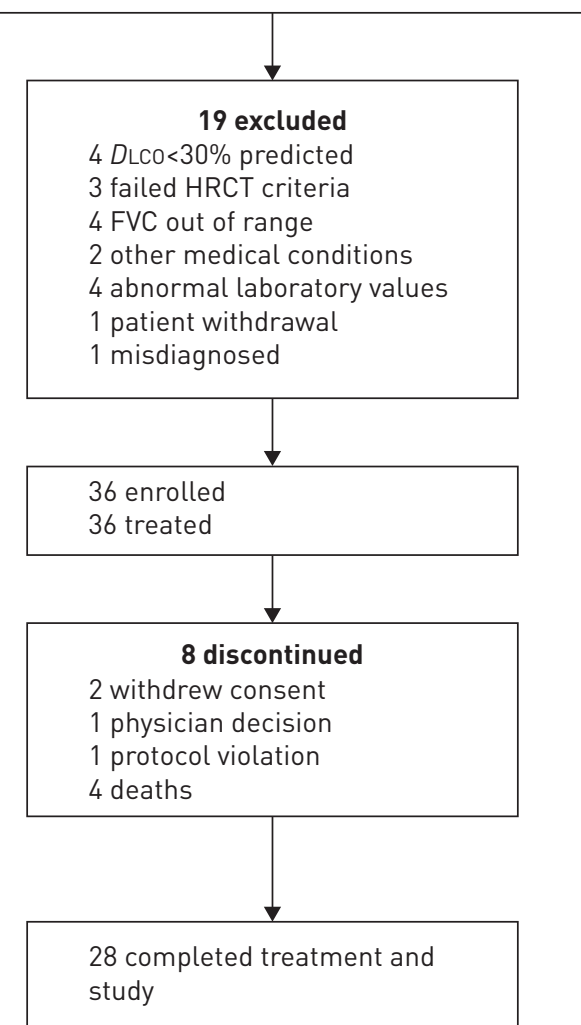

FIGURE 1 Enrolment and outcomes. IPF: idiopathic pulmonary fibrosis; FVC: forced vital capacity; DLco: diffusing capacity of the lung for carbon monoxide; HRCT: high-resolution computed tomography.

\section{TABLE 1 Patient characteristics}

\begin{tabular}{|c|c|c|c|}
\hline & Cohort 1 & Cohort 2 & All \\
\hline Subjects $n$ & 53 & 36 & 89 \\
\hline \multicolumn{4}{|l|}{ Age years } \\
\hline Mean \pm SD & $67.7 \pm 7.0$ & $68.3 \pm 7.2$ & $67.9 \pm 7.0$ \\
\hline Median (range) & $69(47-82)$ & $68.0(49-81)$ & $68.0(47-82)$ \\
\hline Male & $44(83.0)$ & $27(75.0)$ & 71 (79.8) \\
\hline \multicolumn{4}{|l|}{ Race } \\
\hline American Indian & $1(1.9)$ & & $1(1.1)$ \\
\hline Black & & $1(2.8)$ & $1(1.1)$ \\
\hline White & $52(98.1)$ & 35 (97.2) & 87 (97.8) \\
\hline \multicolumn{4}{|l|}{ Height $\mathrm{cm}$} \\
\hline Mean $\pm S D$ & $173.8 \pm 8.2$ & $172.8 \pm 9.6$ & $173.4 \pm 8.8$ \\
\hline Median (range) & $175.3(152-193)$ & $175.3(155-189)$ & $175.3(152-193)$ \\
\hline \multicolumn{4}{|l|}{ Weight kg } \\
\hline Mean \pm SD & $89.4 \pm 14.8$ & $89.6 \pm 15.1$ & $89.5 \pm 14.8$ \\
\hline Median (range) & $89.1(57-122)$ & $89.0(63-122)$ & $89.1(57-122)$ \\
\hline \multicolumn{4}{|c|}{ Subjects with time since IPF diagnosis of } \\
\hline$<1$ year & 19 (35.8) & $15(41.7)$ & 34 (38.2) \\
\hline $1-3$ years & $21(39.6)$ & 12 (33.3) & $33(37.1)$ \\
\hline$>3$ years & $13(24.5)$ & 9 (25) & 22 (24.7) \\
\hline
\end{tabular}

Data are presented as $\mathrm{n}(\%)$ unless otherwise stated. IPF: idiopathic pulmonary fibrosis. 
TABLE 2 Pulmonary function test results

\begin{tabular}{|c|c|c|c|c|c|c|c|c|c|}
\hline & \multicolumn{3}{|c|}{ Cohort 1} & \multicolumn{3}{|c|}{ Cohort 2} & \multicolumn{3}{|c|}{ All } \\
\hline & \multirow[t]{2}{*}{$\mathrm{BL}$} & \multicolumn{2}{|c|}{ Change from BL } & \multirow[t]{2}{*}{ BL } & \multicolumn{2}{|c|}{ Change from BL } & \multirow[t]{2}{*}{ BL } & \multicolumn{2}{|c|}{ Change from $B L$} \\
\hline & & 24 weeks & 48 weeks & & 24 weeks & 48 weeks & & 24 weeks & 48 weeks \\
\hline \multicolumn{10}{|l|}{ FVC } \\
\hline \multicolumn{9}{|l|}{ FVC L } & 66 \\
\hline Mean \pm SE & $2.60 \pm 0.10$ & $-0.08 \pm 0.03$ & $-0.15 \pm 0.04$ & $2.93 \pm 0.16$ & $-0.07 \pm 0.05$ & $-0.13 \pm 0.06$ & $2.73 \pm 0.08$ & $-0.08 \pm 0.02$ & $-0.14 \pm 0.04$ \\
\hline Median (range) & $2.37(1.50-4.22)$ & $-0.08(-0.54-0.36)$ & $-0.17(-0.68-0.57)$ & $2.62(1.32-5.51)$ & $-0.08(-0.37-0.82)$ & $-0.11(-0.75-0.64)$ & $2.53(1.32-5.51)$ & $-0.08(-0.54-0.82)$ & $-0.14(-0.75-0.64)$ \\
\hline \multicolumn{10}{|c|}{ 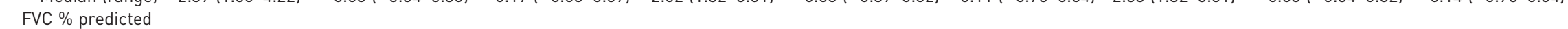 } \\
\hline Mean \pm SE & $62.8 \pm 1.80$ & $-1.80 \pm 0.65$ & $-3.00 \pm 1.04$ & $72.7 \pm 2.69$ & $-1.71 \pm 1.15$ & $-2.25 \pm 1.34$ & $66.8 \pm 1.56$ & $-1.77 \pm 0.60$ & $-2.69 \pm 0.82$ \\
\hline Median (range) & $63.2(42.6-89.6)$ & $-1.54(-15.7-6.8)$ & $-3.62(-15.1-10.7)$ & $69.4(52.9-111.7)$ & $-1.55(-13.2-17.3)$ & $-1.89(-16.7-14.1)$ & $65.9(42.6-111.7)$ & $-1.54(-15.7-17.3)$ & $-3.30(-16.7-14.1)$ \\
\hline \multicolumn{10}{|l|}{$\begin{array}{c}\text { D.co \% predicted, } \\
\text { Hb corrected }\end{array}$} \\
\hline Subjects n & 53 & 43 & 37 & 36 & 29 & 27 & 89 & 72 & 64 \\
\hline Mean \pm SE & $48.3 \pm 1.7$ & $-4.71 \pm 0.88$ & $-4.50 \pm 0.96$ & $49.7 \pm 2.4$ & $-2.01 \pm 0.99$ & $-5.61 \pm 1.08$ & $49.8 \pm 1.41$ & $-3.62 \pm 0.67$ & $-4.9 \pm 0.72$ \\
\hline Median (range) & $47.0(31.1-94.5)$ & $-4.55(-19.7-7.4)$ & $-3.50(-19.0-11.6)$ & $49.5(30.4-89.6)$ & $-3.24(-11.1-10.1)$ & $-4.91(-20.5-4.4)$ & $47.4(30.4-94.5)$ & $-3.68(-19.7-10.1)$ & $-4.71(-20.5-11.6)$ \\
\hline \multicolumn{10}{|l|}{ FEV $1 \%$ predicted } \\
\hline Subjects $\mathrm{n}$ & 53 & 45 & 38 & 36 & 30 & 28 & 89 & 75 & 66 \\
\hline Mean \pm SE & $69.4 \pm 1.80$ & $-2.36 \pm 0.76$ & $-3.44 \pm 1.08$ & $79.7 \pm 2.54$ & $-1.43 \pm 1.33$ & $-1.65 \pm 1.32$ & $73.6 \pm 1.57$ & $-1.99 \pm 0.70$ & $-2.68 \pm 0.84$ \\
\hline Median (range) & $72.5(47.5-103.0)$ & $-2.28(-15.4-9.7)$ & $-3.65(-17.0-10.8)$ & $77.9(60.5-115.4)$ & $-2.36(-19.2-19.4)$ & $-1.59(-15.6-17.7)$ & $74.4(47.5-115.4)$ & $-2.35(-19.2-19.4)$ & $-2.16(-17.0-17.7)$ \\
\hline \multicolumn{10}{|c|}{ (1.0 } \\
\hline Subjects $\mathrm{n}$ & 53 & 43 & 37 & 36 & 30 & 28 & 89 & 73 & 65 \\
\hline Mean \pm SE & $65.2 \pm 1.6$ & $-1.55 \pm 1.21$ & $-2.89 \pm 1.20$ & $71.6 \pm 2.5$ & $-1.86 \pm 0.92$ & $-4.32 \pm 1.16$ & $67.8 \pm 1.41$ & $-1.68 \pm 0.80$ & $-3.51 \pm 0.84$ \\
\hline Median (range) & $64.3(43.1-98.9)$ & $-1.04(-21.9-13.8)$ & $-3.92 \mid-15.0-17.9)$ & $68.9(53.3-118.5)$ & $-1.93(-15.5-9.7)$ & $-4.23(-18.3-8.9)$ & $65.3(43.1-118.5)$ & $-1.79(-21.9-13.8)$ & $-4.02(-18.3-17.9)$ \\
\hline
\end{tabular}


TABLE 3 Quantitative high-resolution computed tomography results for whole-lung fibrosis

\begin{tabular}{|c|c|c|c|c|c|c|c|c|c|}
\hline & \multicolumn{3}{|c|}{ Cohort 1} & \multicolumn{3}{|c|}{ Cohort 2} & \multicolumn{3}{|c|}{ All } \\
\hline & \multirow[t]{2}{*}{$B L$} & \multicolumn{2}{|c|}{ Change from BL } & \multirow[t]{2}{*}{ BL } & \multicolumn{2}{|c|}{ Change from BL } & \multirow[t]{2}{*}{ BL } & \multicolumn{2}{|c|}{ Change from BL } \\
\hline & & 24 weeks & 48 weeks & & 24 weeks & 48 weeks & & 24 weeks & 48 weeks \\
\hline \multicolumn{10}{|l|}{ QLF $\%$} \\
\hline Mean \pm SE & $20.2 \pm 1.6$ & $2.46 \pm 0.94$ & $3.59 \pm 1.08$ & $18.8 \pm 1.8$ & $2.30 \pm 1.10$ & $3.75 \pm 1.42$ & $19.6 \pm 1.2$ & $2.39 \pm 0.71$ & $3.66 \pm 0.86$ \\
\hline Median (range) & $19.0(2-51)$ & $1.0(-10-26)$ & $2.0(-6-27)$ & $17.0(2-44)$ & $2.0(-10-19)$ & $2.0(-13-26)$ & $18.0(2-51)$ & $1.5(-10-26)$ & $2.0(-13-27)$ \\
\hline \multicolumn{10}{|l|}{ GG \% } \\
\hline Mean \pm SE & $18.3 \pm 1.1$ & $-0.80 \pm 0.71$ & $-0.90 \pm 0.82$ & $18.0 \pm 1.3$ & $0.73 \pm 0.78$ & $1.71 \pm 0.72$ & $18.2 \pm 0.8$ & $-0.20 \pm 0.53$ & $0.19 \pm 0.58$ \\
\hline Median (range) & $18.0(5-42)$ & $0.0(-13-8)$ & $0.0(-12-12)$ & $17.5(7-33)$ & $1.0(-10-10)$ & $1.0(-6-10)$ & $18.0(5-42)$ & $0.0(-13-10)$ & $0.0(-12-12)$ \\
\hline \multicolumn{10}{|l|}{ HC \% } \\
\hline Mean \pm SE & $3.9 \pm 1.1$ & $-0.17 \pm 0.42$ & $-0.26 \pm 0.26$ & $2.3 \pm 0.52$ & $0.20 \pm 0.35$ & $-0.61 \pm 0.29$ & $3.3 \pm 0.67$ & $-0.03 \pm 0.29$ & $-0.40 \pm 0.20$ \\
\hline Median (range) & $1.0(0.0-37)$ & $0.0(-13-7)$ & $0.0(-5-5)$ & $1.0(0-13)$ & $0.0(-5-7)$ & $0.0(-7-1)$ & $1.0(0-37)$ & $0.0(-13-7)$ & $0.0(-7-5)$ \\
\hline \multicolumn{10}{|l|}{ QILD score } \\
\hline Mean \pm SE & $42.4 \pm 2.5$ & $1.48 \pm 1.25$ & $2.44 \pm 1.61$ & $39.0 \pm 2.9$ & $3.23 \pm 1.69$ & $4.86 \pm 1.76$ & $41.0 \pm 1.9$ & $2.17 \pm 1.01$ & $3.45 \pm 1.19$ \\
\hline Median (range) & 44.0 (9-93) & $1.0(-19-26)$ & $2.0(-16-28)$ & $37.0(11-74)$ & $3.5(-16-31)$ & $3.5(-22-22)$ & $39.0(9-85)$ & $1.5(-19-31)$ & $3.0(-22-28)$ \\
\hline \multicolumn{10}{|l|}{ TLC mL } \\
\hline Mean \pm SE & $3673 \pm 119$ & $-64 \pm 61$ & $-190 \pm 76$ & $4020 \pm 168$ & $-129 \pm 81$ & $-133 \pm 91$ & $3813 \pm 99$ & $-89 \pm 48$ & $-166 \pm 58$ \\
\hline Median (range) & $3513(2421-6485)$ & $-120(-966-1423)$ & $-161(-1380-889)$ & 3741 (2398-6422) & $-145(-1410-875)$ & $-142(-1002-1467)$ & 3565 (2398-6485) & $-135(-1410-1423)$ & $-158(-1380-1467)$ \\
\hline
\end{tabular}




\begin{tabular}{|c|c|c|c|}
\hline & Cohort 1 & Cohort 2 & Total n (\%) \\
\hline TEAEs reported in $>10 \%$ of subjects & 45 & 33 & $78(87.6)$ \\
\hline Cough & 19 & 9 & $28(31.5)$ \\
\hline Fatigue & 8 & 10 & 18 (20.2) \\
\hline Dyspnoea & 11 & 5 & $16(18.0)$ \\
\hline Upper respiratory tract infection & 9 & 5 & $14(15.7)$ \\
\hline Nasopharyngitis & 7 & 6 & $13(14.6)$ \\
\hline Bronchitis & 6 & 6 & 12 (13.5) \\
\hline Headache & 8 & 3 & $11(12.4)$ \\
\hline Peripheral oedema & 4 & 6 & $10(11.2)$ \\
\hline Diarrhoea & 7 & 3 & $9(10.1)$ \\
\hline Dizziness & 5 & 4 & $10(11.2)$ \\
\hline Nausea & 6 & 4 & $10(11.2)$ \\
\hline Urinary tract infection & 5 & 4 & $9(10.1)$ \\
\hline Pneumonia & 6 & 2 & $8(9.0)$ \\
\hline Idiopathic pulmonary fibrosis exacerbation & 3 & 5 & $8(9.0)$ \\
\hline Nasal congestion & 1 & 7 & $8(9.0)$ \\
\hline Treatment-emergent serious adverse events" & 13 & 11 & $24(27.0)$ \\
\hline Cardiac disorders & 0 & 4 & $4(4.5)$ \\
\hline Gastrointestinal disorders & 1 & 0 & $1(1.1)$ \\
\hline Infections and infestations & 7 & 3 & $10(11.2)$ \\
\hline Pneumonia & 6 & 2 & $8(9.0)$ \\
\hline Influenza & 0 & 1 & $1(1.1)$ \\
\hline Neoplasms & 0 & 1 & $1(1.1)$ \\
\hline Nervous system disorders & 2 & 0 & $2(2.2)$ \\
\hline Psychiatric disorders & 1 & 0 & $1(1.1)$ \\
\hline Renal and urinary disorders & 0 & 1 & $1(1.1)$ \\
\hline Respiratory, thoracic and mediastinal disorders & 5 & 6 & $11(12.4)$ \\
\hline Idiopathic pulmonary fibrosis & 2 & 4 & $6(6.7)$ \\
\hline Acute respiratory failure & 2 & 0 & $2(2.2)$ \\
\hline Dyspnoea & 0 & 1 & $1(1.1)$ \\
\hline Epistaxis & 0 & 1 & $1(1.1)$ \\
\hline Hypoxia & 0 & 1 & $1(1.1)$ \\
\hline Pulmonary hypertension & 0 & 1 & $1(1.1)$ \\
\hline Respiratory arrest & 1 & 0 & $1(1.1)$ \\
\hline Respiratory failure & 0 & 1 & $1(1.1)$ \\
\hline Vascular disorders & 2 & 0 & $2(2.2)$ \\
\hline
\end{tabular}

TEAEs included medical conditions, signs and symptoms not previously observed in the subject that emerged during the protocol-specified adverse event reporting period. A serious adverse event was defined as any adverse event or suspected adverse reaction that resulted in death, a life-threatening adverse event, inpatient hospitalisation or prolongation of existing hospitalisation, persistent or significant incapacity, or substantial disruption of the ability to conduct normal life functions. " : 24 subjects with serious adverse events; some subjects experienced more than one serious adverse events. " : benign, malignant and unspecified, including cysts and polyps.

respiratory distress syndrome/sepsis secondary to colon cancer surgery. None of the deaths was considered by the investigator to be related to the study drug. No National Cancer Institute grade 3 or 4 laboratory abnormalities [13] were reported from the Safety Population. No infusions were stopped due to safety concerns. There were no obvious differences in AEs or SAEs between the two cohorts.

\section{Outcomes}

Results for pulmonary function outcomes are shown in table 2 and summarised in table 5, and quantitative HRCT outcomes are shown in table 3. Tables 2, 3 and 5 show mean baseline values and changes from baseline for each cohort individually as well as for pooled results. Table 5 shows the change of FVC, FVC \% predicted, DLCO \% predicted and TLC \% predicted from BL to week 48 for each cohort, and within each cohort for all subjects, for those with BL FVC $\geqslant 55 \%$ predicted and for those with BL FVC $<55 \%$ predicted. Table 5 also shows the number of subjects that showed substantial negative changes in FVC $\%$ predicted $(\leqslant-10 \%)$ and positive changes FVC $\%$ predicted $(>0 \%)$ at week 48.

Mean \pm SEM observed FVC change for the combined cohorts 1 and 2 was $-140 \pm 35 \mathrm{~mL}(-2.7 \%$ predicted $)$ from BL to week 48 for the entire study population $(n=89$ at BL and $n=66$ at week 48 ) and $-110 \pm 37 \mathrm{~mL}$ 
TABLE 5 Summary of mean pulmonary function changes $(\Delta)$ at week 48

\begin{tabular}{|c|c|c|c|c|c|c|c|c|c|c|c|}
\hline \multirow[t]{2}{*}{ Cohort } & \multirow{2}{*}{$\begin{array}{l}\text { Subjects } \\
\text { at BL n }\end{array}$} & \multicolumn{2}{|c|}{ FVC L } & \multicolumn{2}{|c|}{ FVC $\%$ predicted } & \multicolumn{2}{|c|}{ D.co $\%$ predicted } & \multicolumn{2}{|c|}{ TLC $\%$ predicted } & \multirow{2}{*}{$\begin{array}{c}\Delta \mathrm{FVC} \\
\leqslant-10 \% \\
\text { predicted }\end{array}$} & \multirow{2}{*}{$\begin{array}{l}\Delta \mathrm{FVC}>0 \% \\
\text { predicted }\end{array}$} \\
\hline & & $\begin{array}{c}\text { Subjects } \\
n\end{array}$ & $\begin{array}{c}\Delta \mathrm{FVC} \\
\mathrm{L}\end{array}$ & $\begin{array}{c}\text { Subjects } \\
n\end{array}$ & $\begin{array}{c}\Delta \mathrm{FVC} \% \\
\text { predicted }\end{array}$ & $\begin{array}{c}\text { Subjects } \\
n\end{array}$ & $\begin{array}{l}\Delta D\llcorner c o \% \\
\text { predicted }\end{array}$ & $\begin{array}{c}\text { Subjects } \\
n\end{array}$ & $\begin{array}{c}\Delta \mathrm{TLC} \% \\
\text { predicted }\end{array}$ & & \\
\hline $\begin{array}{l}\mathrm{BL} F \mathrm{FV} \geqslant 55 \% \\
\text { predicted }\end{array}$ & 38 & 33 & -0.12 & 33 & -2.19 & 33 & -3.43 & 31 & -2.22 & $4(12.1)$ & $9(27.3)$ \\
\hline $\begin{array}{c}\text { BL FVC }<55 \% \\
\text { predicted }\end{array}$ & 15 & 5 & -0.37 & 5 & -8.42 & 4 & -12.92 & 5 & -8.08 & $1(20.0)$ & $0(0.0)$ \\
\hline $\begin{array}{c}\text { BL FVC } \geqslant 55 \% \\
\text { predicted }\end{array}$ & 32 & 27 & -0.11 & 27 & -1.93 & 27 & -5.61 & 27 & -4.00 & $3(11.1)$ & 10 (37.0) \\
\hline $\begin{array}{c}\text { BL FVC }<55 \% \\
\text { predicted }\end{array}$ & 4 & 1 & -0.58 & 1 & -11.01 & 0 & 0.0 & 1 & -12.80 & $1(100.0)$ & $0(0.0)$ \\
\hline Cohort 1+2 & 89 & 66 & -0.14 & 66 & -2.69 & 64 & -4.94 & 64 & -3.51 & $9(13.6)$ & 19 (28.8) \\
\hline $\begin{array}{c}\text { BL FVC } \geqslant 55 \% \\
\text { predicted }\end{array}$ & 70 & 60 & -0.11 & 60 & -2.07 & 60 & -4.41 & 58 & -3.05 & $7(11.7)$ & 19 (31.7) \\
\hline
\end{tabular}

Data are presented as $\mathrm{n}(\%)$ unless otherwise stated. BL: baseline; FVC: forced vital capacity; DLco: diffusing capacity of the lung for carbon monoxide; TLC: total lung capacity.

$(-2.1 \%$ predicted) in subjects with $\mathrm{BL}$ FVC $>55 \%$ predicted $(\mathrm{n}=70$ at $\mathrm{BL}$ and $\mathrm{n}=60$ at week 48$)$. At week 48, 19 (28.8\%) had no decline in FVC \% predicted. In contrast, nine (13.6\%) subjects had an absolute decline in FVC $\%$ predicted $\geqslant 10 \%$. Using imputation for all subjects with at least one post-BL determination, the mean FVC changes at week 48 were $-205 \pm 33 \mathrm{~mL}$ (5.5\% predicted) for all subjects $(\mathrm{n}=82)$ and $-128 \pm 35 \mathrm{~mL}(2.8 \%$ predicted $)$ for subjects with BL FVC $\geqslant 55 \%$ predicted $(\mathrm{n}=65)$.

Extent and change of pulmonary fibrosis was assessed using quantitative HRCT. HRCT scores at BL, Week 24 and week 48 are summarised in table 3 by cohort and combined. In the combined results for cohorts 1 and 2, the BL QLF was $19.6 \%$ and the mean change at week 48 was $+3.66 \%$. For GG, the baseline extent was $18.2 \%$ and the mean change at week 48 was $+0.19 \%$. There was less HC at baseline (3.3\%) and a mean change of $-0.4 \%$ at week 48 . The greatest magnitude of change at week 48 , both positive and negative, occurred in QLF. While the majority of patients (65\%) exhibited an increase in reticular fibrosis, 35\% exhibited stable or improved reticular fibrosis at week 48. Regardless of the analysis method, observed or imputed, the same 16 subjects showed improved fibrosis $(20 \%$ of full analysis set, $24 \%$ of completers) and seven showed stable fibrosis (9\% of full analysis set, $11 \%$ of completers).

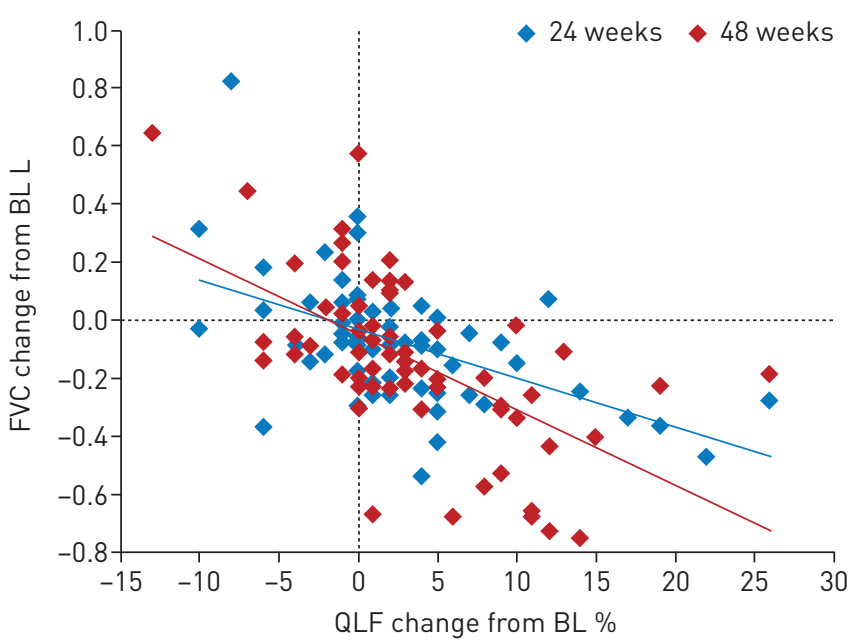

FIGURE 2 Correlations between fibrosis (QLF) change and forced vital capacity (FVC) change with all available data from baseline (BL) at week $24(n=74)$ and week $48(n=66)$. 


\begin{tabular}{|c|c|c|c|c|}
\hline $\begin{array}{l}\text { Fibrosis } \\
\text { subtype }\end{array}$ & Week & Subjects $n$ & Pearson's r & p-value \\
\hline \multirow[t]{2}{*}{ QLF } & 24 & 74 & -0.520 & $<0.0001$ \\
\hline & 48 & 66 & -0.624 & $<0.001$ \\
\hline GG & 48 & 66 & -0.233 & $0.074^{\#}$ \\
\hline QILD & 48 & 66 & -0.514 & $<0.001$ \\
\hline
\end{tabular}

QLF: reticular fibrosis with architectural distortion; GG: ground glass; QILD: QLF+GG+honeycombing.

\#: nonsignificant.

Figure 2 and table 6 show the relationships between change from BL in FVC and QLF at weeks 24 and 48. An inverse correlation is noted between changes in the extent of QLF and changes in FVC, $(r=-0.520$ and $\mathrm{r}=-0.624, \mathrm{p}<0.001)$ at both 24 and 48 weeks. To further characterise the relationship between apparent changes in fibrosis and changes in FVC, a post hoc analysis was performed whereby FVC changes in subjects that completed the week 24 and week 48 HRCT assessments were categorised by QLF change from $\mathrm{BL}$ at weeks 24 and 48 (figure 3) since those were the only time-points at which changes in both FVC and HRCT values could be assessed. Subjects with QLF change from BL $>0$ had a mean FVC change from BL at week 48 of $-0.24 \mathrm{~L}$. However, those with QLF change from $\mathrm{BL} \leqslant 0$ had a positive average FVC change from $\mathrm{BL}$, and at Week 48, the average FVC change from BL for these subjects was $+0.04 \mathrm{~L}$.

Patient reported outcomes were assessed using the St George's Respiratory Questionnaire and the University of California at San Diego Shortness of Breath Questionnaire (table 7). None of the outcomes was considered to reflect a significant change from $\mathrm{BL}$.

We evaluated demographic properties, pulmonary function, quantitative fibrosis parameters at BL and plasma CTGF levels to determine if any BL factor or combination of BL factors could identify those patients who exhibited positive or negative changes in FVC or QLF; however, no BL property was found to be a reliable predictor or correlate of outcome.

\section{Discussion}

This study was initiated after observing reversal of established pulmonary fibrosis by FG-3019 in a mouse model of radiation-induced pulmonary fibrosis. The aim of this clinical study was to determine the safety and tolerability of FG-3019, and to assess whether modulation of CTGF activity by the antibody could impact established fibrosis in a fulminant human interstitial lung disease such as IPF. Two doses of the monoclonal antibody administered to IPF patients by intravenous infusion every 3 weeks for 45 weeks were well tolerated in the patients enrolled in this study. AEs were generally mild and the SAEs were typical of those seen in subjects with IPF. A wide range of disease severity was enrolled in the trial, with FVC values

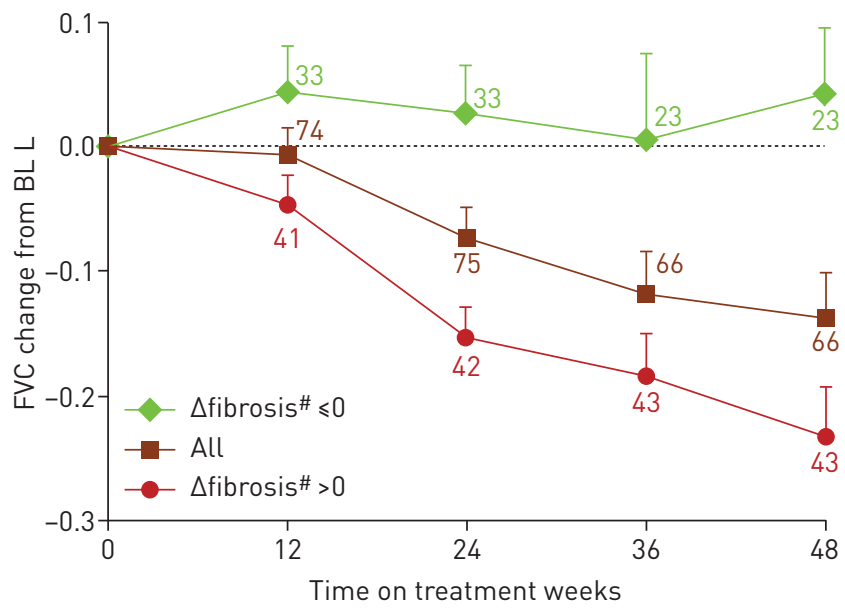

FIGURE 3 Categorical changes in forced vital capacity (FVC) based on reticular fibrosis with architectural distortion (QLF) change $(\Delta)$ from baseline (BL) at week 24 and week 48. " : change in QLF score from BL to week 24 and week 48; categorical FVC changes at weeks 12 and 24 were based on week 24 change in fibrosis, and categorical FVC change at weeks 36 and 48 were based on week 48 change in fibrosis. 
TABLE 7 Patient-reported outcomes: observed values for subjects that completed week 48 assessments

\begin{tabular}{|c|c|c|c|c|c|c|}
\hline & \multicolumn{3}{|c|}{ BL FVC $\geqslant 55 \%$ predicted $^{\#}$} & \multicolumn{3}{|c|}{ All" } \\
\hline & \multirow[t]{2}{*}{ BL } & \multicolumn{2}{|c|}{ Change } & \multirow[t]{2}{*}{ BL } & \multicolumn{2}{|c|}{ Change } \\
\hline & & Week 24 & Week 48 & & Week 24 & Week 48 \\
\hline UCSD-SOBQ total & $29.00 \pm 2.42$ & $0.90 \pm 1.57$ & $1.30 \pm 1.85$ & $29.90 \pm 2.25$ & $1.70 \pm 1.50$ & $2.90 \pm 1.88$ \\
\hline SGRQ symptoms & $49.90 \pm 2.51$ & $1.50 \pm 2.22$ & $0.70 \pm 2.29$ & $51.10 \pm 2.41$ & $1.09 \pm 2.04$ & $0.60 \pm 2.08$ \\
\hline SGRQ activity & $57.20 \pm 2.15$ & $1.60 \pm 2.00$ & $1.80 \pm 1.74$ & $58.70 \pm 2.05$ & $2.10 \pm 1.86$ & $2.40 \pm 1.64$ \\
\hline SGRQ impacts & $30.10 \pm 2.12$ & $0.50 \pm 1.98$ & $0.50 \pm 2.07$ & $32.00 \pm 2.08$ & $0.20 \pm 1.82$ & $0.20 \pm 1.92$ \\
\hline SGRQ total & $42.10 \pm 1.79$ & $0.70 \pm 1.69$ & $0.80 \pm 1.66$ & $43.70 \pm 1.76$ & $0.80 \pm 1.54$ & $0.80 \pm 1.52$ \\
\hline
\end{tabular}

Data are presented as mean \pm SEM. BL: baseline; FVC: forced vital capacity; UCSD-SOBQ: University of California at San Diego Shortness of Breath Questionnaire; SGRQ: St George's Respiratory Questionnaire. " : n=60; ๆ: n=66.

ranging from $42.6 \%$ to $111.7 \%$ predicted. Patients with FVC values below 55\% predicted generally had higher QLF scores at BL, and demonstrated worsening of both fibrosis and pulmonary function.

The study was designed to be open-label and thus did not include a placebo group. FG-3019-treated patients in this study showed an average decline of $140 \mathrm{~mL}$ over the treatment period of 48 weeks. Of the subjects who completed a 48-week course of treatment, $13.6 \%$ experienced a decline of FVC \% predicted of $\geqslant 10 \%$. In contrast, $30 \%$ of treated subjects showed an increase of FVC (range $0.2-14.1 \%$ predicted).

A subset of subjects exhibited reduced reticular fibrosis 48 weeks after initiating dosing with FG-3019. Changes in fibrosis score correlated with changes in pulmonary function. In an analysis of the FVC outcomes based on the changes in fibrosis, subjects with stable or reduced fibrosis (QLF change from BL $\leqslant 0)$ had a positive average FVC change from BL at week 48 of $+40 \mathrm{~mL}$. In contrast, subjects with increased fibrosis (QLF change from BL >0) had a mean FVC change from BL at week 48 of $-240 \mathrm{~mL}$. Quantifying pulmonary fibrosis by computer algorithms has greater sensitivity for identifying changes in fibrosis than visual scoring [14]. Consequently, some of the changes seen in pulmonary fibrosis may represent the variable natural progression of this heterogeneous disease.

Treatment of scleroderma lung disease with cyclophosphamide yielded improvement in quantified lung fibrosis in some subjects with a statistically significant correlation with changes in FVC [11]. However, quantitative change in fibrosis has not been studied longitudinally in IPF patients to enable an expected change in fibrosis in a manner analogous to the predicted changes in FVC. Recent studies have used quantitative HRCT to evaluate changes of fibrosis in untreated IPF subjects. MALDONADO et al. [15] used a computer-aided system for quantifying IPF fibrosis based on unique HRCT texture patterns for GG, QLF and HC, and the changes in those HRCT-derived patterns between two time-points 3-15 months apart. They showed that changes in reticular fibrosis and total fibrosis were predictive of survival. ODA et al. [16], using a similar HRCT scoring system approach, showed that HRCT fibrosis scores at 6 and 12 months after diagnosis were significantly increased compared to those observed at the initial diagnosis and that patients with elevated HRCT fibrosis score at 6 months had a poor prognosis. KIM et al. [17] showed that QLF scores closely correlate with disease extent and that 6-month change in QLF is predictive of FVC decline at 18 months, whereas quantitative CT measures based only on measures of lung density (kurtosis) predict mortality but not functional change. Quantitative HRCT has not been previously reported as a measure of outcomes in an interventional IPF clinical trial and has not been established or validated as a surrogate marker of clinically meaningful outcomes. Nonetheless, as noted in a recent position paper [18], the use of quantitative CT algorithms as an end-point in clinical trials is an area of growing interest and promise.

In summary, this open-label study designed to assess the safety and potential for efficacy of long-term use of FG-3019, a novel antifibrotic agent given intravenously for 45 weeks in patients with IPF, demonstrated a good safety profile and yielded encouraging outcomes with regard to changes in pulmonary function and extent of pulmonary fibrosis by imaging. The limitations incurred by the lack of a placebo control group as well as the need to validate the novel computerised scoring system for quantifying the extent of fibrosis in this study is evident. Further studies are warranted to confirm the observed findings, and a randomised placebo-controlled phase 2 clinical trial of FG-3019 in IPF patients is currently underway.

\section{Acknowledgements}

We gratefully acknowledge Loredie Lugos, Joyce Alejo-Stone, Tom Guntly (all FibroGen Inc. San Francisco CA, USA) and Hal Collard (University of California, San Francisco, CA USA) for their operational support of this study. 


\section{References}

1 Raghu G, Collard HR, Egan JJ, et al. An official ATS/ERS/JRS/ALAT statement: idiopathic pulmonary fibrosis: evidence-based guidelines for diagnosis and management. Am J Respir Crit Care Med 2011; 183: 788-824.

2 Kim DS, Collard HR, King TE Jr. Classification and natural history of the idiopathic interstitial pneumonias. Proc Am Thorac Soc 2006; 3: 285-292.

3 Lipson KE, Wong $\mathrm{C}$, Teng Y, et al. CTGF is a central mediator of tissue remodeling and fibrosis and its inhibition can reverse the process of fibrosis. Fibrogenesis Tissue Repair 2012; 5: Suppl. 1, S24.

4 Mori T, Kawara S, Shinozaki M, et al. Role and interaction of connective tissue growth factor with transforming growth factor-beta in persistent fibrosis: A mouse fibrosis model. J Cell Physiol 1999; 181: 153-159.

5 Wang Q, Usinger W, Nichols B, et al. Cooperative interaction of CTGF and TGF-beta in animal models of fibrotic disease. Fibrogenesis Tissue Repair 2011; 4: 4

6 Lipson KE, Wirkner U, Sternlicht M, et al. Rapid reversal of radiation-induced murine pneumonitis by treatment with the anti-CTGF monoclonal antibody FG-3019. Eur Respir J 2011; 38: Suppl. 55, P668.

7 Mageto Y, Flaherty K, Brown K, et al. Safety and tolerability of human monoclonal antibody FG-3019, Anti-connective tissue growth factor, in patients with idiopathic pulmonary fibrosis. Chest 2004; 126: Suppl., 773S-a.

8 Chong D, Brown MS, Kim HJ, et al. Reproducibility of volume and densitometric measures of emphysema on repeat computed tomography with an interval of 1 week. Eur Radiol 2012; 22: 287-294.

9 Miller MR, Crapo R, Hankinson J, et al. General considerations for lung function testing. Eur Respir J 2005; 26 : $153-161$.

10 Brown MS, Kim HJ, Abtin F, et al. Reproducibility of lung and lobar volume measurements using computed tomography. Acad Radiol 2010; 17: 316-322.

11 Kim HJ, Brown MS, Elashoff R, et al. Quantitative texture-based assessment of one-year changes in fibrotic reticular patterns on HRCT in scleroderma lung disease treated with oral cyclophosphamide. Eur Radiol 2011; 21: $2455-2465$

12 Kim HJ, Li G, Gjertson D, et al. Classification of parenchymal abnormality in scleroderma lung using a novel approach to denoise images collected via a multicenter study. Acad Radiol 2008; 15: 1004-1016.

13 US Department of Health and Human Services. Common Terminology Criteria for Adverse Events (CTCAE). http://evs.nci.nih.gov/ftp1/CTCAE/CTCAE_4.03_2010-06-14_QuickReference_8.5x11.pdf Date last updated: June 14,2010

14 Best AC, Meng J, Lynch AM, et al. Idiopathic pulmonary fibrosis: physiologic tests, quantitative CT indexes, and CT visual scores as predictors of mortality. Radiology 2008; 246: 935-940.

15 Maldonado F, Moua T, Rajagopalan S, et al. Automated quantification of radiological patterns predicts survival in idiopathic pulmonary fibrosis. Eur Respir J 2014; 43: 204-212.

16 Oda $\mathrm{K}$, Ishimoto $\mathrm{H}$, Yatera $\mathrm{K}$, et al. High-resolution CT scoring system-based grading scale predicts the clinical outcomes in patients with idiopathic pulmonary fibrosis. Respir Res 2014; 15: 10.

17 Kim HJ, Brown MS, Chong D, et al. Comparison of the quantitative CT imaging biomarkers of idiopathic pulmonary fibrosis at baseline and early change with an interval of 7 months. Acad Radiol 2015; 22: 70-80.

18 Hansell DM, Goldin JG, King TE, et al. CT staging and monitoring of fibrotic interstitial lung diseases in clinical practice and treatment trials: a position paper from the Fleischner Society. Lancet Respir Med 2015; 3: 483-496. 